\title{
Determinants and Incidence of Chronic Kidney Disease on Tenofovir-Based Antiretroviral Therapy Regimens: A Cohort Study in HIV-Infected Adults in South China
}

\section{Fang Liu}

Xixi Hospital of Hangzhou https://orcid.org/0000-0002-5379-8816

Hong Liu

Xixi Hospital of Hangzhou

\section{Chen Chen}

Temple University

Liang-bin Miao

Xixi Hospital of Hangzhou

Zhao-yi Li

Xixi Hospital of Hangzhou

\section{Yi Wang}

Xixi Hosptial of Hangzhou

\section{Miao-chan Wang}

Xixi Hospital of Hangzhou

Jin-song Huang

Xixi Hospital of Hangzhou

Jian-feng Bao ( $\sim$ hzxxbjf@outlook.com )

Xixi Hospital of Hangzhou

\section{Research}

Keywords: HIV, Antiviral therapy, Chronic kidney disease, Tenofovir, China

Posted Date: March 31st, 2021

DOl: https://doi.org/10.21203/rs.3.rs-365588/v1

License: (1) This work is licensed under a Creative Commons Attribution 4.0 International License.

Read Full License 


\section{Abstract}

Background: To evaluate the incidence and risk factors of stage 3 chronic kidney disease (CKD) and rapid kidney function decline (RKFD) among Chinese HIV-1 infected patients starting with tenofovir (TDF)based regimen.

Methods: We enrolled in 797 TDF-initiated HIV-1-infected patients in a Chinese cohort. Kidney dysfunction were defined as stage 3 CKD (eGFR $<60 \mathrm{~mL} / \mathrm{min} / 1.73 \mathrm{~m}^{2}$ during follow-up) and RKFD (eGFR decline $>10$ $\mathrm{mL} / \mathrm{min} / 1.73 \mathrm{~m}^{2} /$ year). A linear mixed-effects model was used to quantify the average eGFR change per 48 weeks. A generalized estimating equation regression analysis was conducted to determine the risk factors associated with renal dysfunction. The method of multiple imputation was used to reduce bias caused by missing data.

Results: In this retrospective study, 14 (2\%) patients experienced stage 3 CKD, and 272 (34\%) individuals experienced RKFD during a median of 26 (IQR, 4-78; maximum 325) weeks follow-up period. The mean loss in eGFR per 48 weeks increased consistently over time, from $-2.59 \mathrm{~mL} / \mathrm{min} / 1.73 \mathrm{~m}^{2}$ before 48 weeks to $-17.61 \mathrm{~mL} / \mathrm{min} / 1.73 \mathrm{~m}^{2}$ after 288 weeks. For every $10 \mathrm{~mL} / \mathrm{min} / 1.73 \mathrm{~m}^{2}$ increase of eGFR, the risk of RKFD increased by $29 \%(95 \% \mathrm{Cl}: 18 \%, 40 \%)$. Each 10 years older and every $10 \mathrm{~mL} / \mathrm{min} / 1.73 \mathrm{~m}^{2}$ higher in baseline eGFR, the risk of stage 3 CKD increased to 1.56 (95\% Cl: $1.00,2.43)$ and decreased by $65 \%(95 \%$ Cl: $48 \%, 76 \%$ ), respectively. Anemia and higher viral load were significantly associated with RKFD. The results were robust across a range of multiple imputation analysis.

Conclusions: TDF-associated CKD is rare in HIV-1 infected Chinese adults. Longer TDF-exposed patients are more likely to have renal dysfunction, especially those with older age, anemia, lower baseline eGFR and higher viral load.

\section{Introduction}

The widespread use of combination antiretroviral therapy (cART) has essentially improved the life expectancy of the human immunodeficiency virus (HIV)-positive individuals [1]. The need to manage noncommunicable diseases (NCDs) becomes evident with the advent of ART [2]. Chronic kidney disease (CKD) is one of the important NCDs associated with morbidity and mortality [3, 4]. Since HIV- 1 infection is currently incurable and requires lifelong treatment, maintaining kidney function is especially important for HIV-1 infected patients. In addition, kidney function has been reported to deteriorate faster in HIV-1 infected patients than in the general population $[5,6]$.

Tenofovir disoproxil fumarate (TDF), a reverse transcriptase inhibitor, which was recommended as one of the antiretrovirals (ARVs) at first-line therapy by World Health Organization (WHO), is currently the most valuable drug against HIV. However, TDF has clinically significant nephrotoxicity [7, 8]. Patients in Asia are more susceptible to nephrotoxic ARVs, because of their lower body weight than whites or blacks [9, 10]. HIV-1 infected patients in China live longer due to more and more abundant clinical resources, which 
further underscores the importance of maintaining these patients' kidney function. At present, only one study in China that included comorbidity status and information on kidney function at baseline but limited to patients $<50$ years of age, has assessed the prevalence of and factors associated with renal dysfunction in TDF users infected with HIV-1[11]. However, the small sample size (269 patients) and short-term observation (168 weeks) in this report limits its generalizability.

From the above points, we conducted a retrospective study in a Chinese cohort of treatment naïve HIV-1positive individuals who initiated TDF-containing regimens between Jan., 2012 and Jun., 2018. The objective of this study was to describe the incidence and risk factors of renal dysfunction in HIV-1 infected patients receiving TDF in a "real world" clinical setting in the largest infectious disease hospital in South China.

\section{Methods}

\section{Study Population}

This is a retrospective, observational cohort study conducted at the Infectious Diseases Department (IDD) at Xixi Hospital (Hangzhou, Zhejiang, South China). All treatment naïve HIV-1-positive patients with records of TDF initiation between January 10, 2012 and June 25, 2018 were screened for eligibility. A total of 900 patients were enrolled as of December 30,2018. This study was approved by the Institutional Review Board of Xixi Hospital.

\section{Data Collection and Inclusion Criteria}

Data extracted from the IDD database included demographic parameters, date of TDF initiation, details of the cART regimens, route of HIV-1 transmission, comorbidities, AIDS diagnosis days and laboratory variables (HIV-1 RNA viral load, CD 4 count, and serum creatinine [SCr]) at 2 weeks, 1 month, 2 months, 3 months, and every 3 months thereafter until December, 2018. Baseline was defined as the date of starting CART. Each enrolled patient was 17 years old or more, had at least one additional eGFR measurement since January, 2012. The flowchart was detailed in Fig.1.

[Insert Fig.1]

\section{Quantitative Variables}

The three-variable Modification of Diet in Renal Disease (MDRD) formula adjusted for Chinese populations was used to calculate the eGFR values: eGFR=234.96× [serum creatinine] ${ }^{-0.926 \times[a g e]}$ $-0.280 \times[0.828$ for females]. This equation performs better than the Chronic Kidney Disease Epidemiology Collaboration (CKD-EPI) equation in Chinese patients $[12,13]$.

Patients who took TDF alone or any TDF-containing regimen (TDF + lamivudine [3TC], or emtricitabine $[$ FTC] + nevirapine [NVP], or efavirenz [EFV], or zidovudine [AZT]) were classified as TDF-receiving users. 
The two outcome definitions of this study were stage-3 CKD (eGFR $\geq 60 \mathrm{~mL} / \mathrm{min} / 1.73 \mathrm{~m}^{2}$ at baseline and eGFR $<60 \mathrm{~mL} / \mathrm{min} / 1.73 \mathrm{~m}^{2}$ during follow-up), and rapid kidney function decline (RKFD: with progression to CKD; eGFR decline $>10 \mathrm{~mL} / \mathrm{min} / 1.73 \mathrm{~m}^{2} /$ year, estimated by least squares regression).

\section{Statistical Analyses}

A linear mixed-effects regression model based on a Generalized Estimate Equation (GEE), with random intercepts, was applied to quantify the average change per 48 weeks of eGFR during different periods on CART. GEE logistic regression was used to analyze the relationship of baseline characteristics with RKFD and with stage 3 CKD. Time to the incident of stage 3 CKD was calculated by the Kaplan-Meier survival curve. We constructed two analyses; one was adjusted for age, gender and body mass index (BMI) to estimate the crude associations of each baseline characteristic with RKFD and with stage 3 CKD; another was performed on a range of multivariable analyses, to determine variables independently correlated with RKFD or associated with stage 3 CKD.

Data on HIV-1 RNA viral load were not available in up to $40 \%$ of patients, so a missing value category was used in the main analyses [14]. In sensitivity analyses, 5 imputed datasets, established by multiple imputation with chained equations, which can reduce bias caused by the exclusion of individuals with missing data of HIV-1 RNA viral load at baseline, were developed and run separately, and the results were combined using Rubin's method [15, 16] (Additional file 1: Table S1-S3).

All analyses were performed using the R software, version 3.3.1 (http://www.R-project.org). A result was considered statistically significant when the two-tailed $P$ value was below 0.05 . The paper is reported according to the Strengthening the Reporting of Observational Studies in Epidemiology (STROBE) statement [17].

\section{Results}

\section{Participant characteristics}

As shown in the flowchart (Fig.1), a total of 900 patients were enrolled, and 797 patients were eligible for participation. Table 1 showed the baseline and clinical characteristics of patients. The median age was 31 (interquartile range [IQR], 26-39) years. Most enrolled patients were male (94.3\%) and were infected via male-male sex. The median follow-up time was 26 (IQR, 4-78; maximum 325) weeks. There were 10218 eGFR measurements and a median of 6 eGFR measurements per person (IQR, 3-11). The median interval between eGFR measurements was 90 (IQR, 30-90) days.

\section{Outcome Data}

In this cohort study, 444 (56\%) patients had negative eGFR slopes, while 353 (44\%) patients had positive eGFR slopes. Of 797 patients, 14 (2\%) experienced stage 3 CKD during a median 54 (IQR, 39,90) weeks follow-up period, and 272 (34\%) experienced RKFD at a median follow- up of 13 (IQR, 3-56) weeks. Fig. 2 
shows the cumulative incidence of stage 3 CKD over a 325-weeks (6.5 years) period, by TDF exposure status. The cumulative incidence of stage 3 CKD increased to $2.3 \%(95 \% \mathrm{Cl}: 0.3 \%, 4.2 \%)$ after 200 weeks of exposure to TDF.

\section{Quantification of Mean Loss in eGFR}

Table 2 shows the eGFR loss on different cART duration among TDF users. The average eGFR change per 48 weeks was -1.29 (95\% Cl: $-1.84,-0.75)$ in the multivariate generalized linear mixed-effects analysis. The adjusted mean loss in eGFR increased consistently over time, from $-2.59 \mathrm{~mL} / \mathrm{min} / 1.73 \mathrm{~m}^{2}$ before 48 weeks to $-17.61 \mathrm{~mL} / \mathrm{min} / 1.73 \mathrm{~m}^{2}$ after 288 weeks. The results were similar in the unadjusted model and sensitivity analysis (Additional file 1: Table S1).

\section{Determinants of Renal dysfunction}

Fig. 3 shows the determinants of RKFD. In adjusted analyses, independently significant factors associated with RKFD were eGFR, anemia and HIV-RNA viral load at baseline. For every $10 \mathrm{~mL} / \mathrm{min} / 1.73$ $\mathrm{m}^{2}$ increase, the aOR of RKFD increased by $29 \%(95 \% \mathrm{Cl}: 18 \%, 40 \%)$. The risk of RKFD increased significantly (aOR, $1.64 ; 95 \% \mathrm{Cl}: 1.07,2.51)$ among patients with anemia. Each $\log _{10}$ viral load higher was associated with a 1.29-fold increased risk of RKFD (aOR, 1.29; 95\% Cl: 1.06, 1.58).

Fig. 4 shows the factors associated with stage 3 CKD. Baseline eGFR and age were significantly associated with the development of stage 3 CKD in the adjusted analysis. For every $10 \mathrm{~mL} / \mathrm{min} / 1.73 \mathrm{~m}{ }^{2}$ increase in baseline eGFR, the risk of stage 3 CKD decreased by 65\% (95\% Cl: 48\%, 76\%). Each 10 years older age was associated with a 1.56-fold increased risk of developing stage 3 CKD (aOR, 1.56; 95\% Cl: $1.00,2.43)$. Sensitivity analyses that were conducted in five imputed datasets did not alter the trends of our study Additional file 1: Table S2, S3).

\section{Discussion}

In this observational cohort study of HIV-1-infected south Chinese patients who initiated a TDFcontaining CART, we quantified the mean loss of eGFR over time and evaluated the determinants of renal dysfunction among patients who initiated TDF-based ART.

The slope of eGFR was negative in most patients, and more than $34 \%$ of individuals developed RKFD, but only $2 \%$ of patients experienced stage 3 CKD. The effect of TDF on the decrement of eGFR was evident, continuously increased during the 6-years observation period (from $-2.59 \mathrm{~mL} / \mathrm{min} / 1.73 \mathrm{~m}^{2}$ before 48 weeks to $-17.61 \mathrm{~mL} / \mathrm{min} / 1.73 \mathrm{~m}^{2}$ after 288 weeks). The independent factors associated with a greater likelihood of RKFD was higher eGFR and HIV-RNA viral loads at baseline. Patients with anemia had an increased risk of RKFD. Older age and lower baseline eGFR were significantly associated with an increased risk of stage 3 CKD. 
Previous studies in Sub-Saharan Africa have shown that renal dysfunction is highly prevalent in HIV-1 infected patients receiving cART. The incidence of stage 3 CKD was estimated to be $5.2-25 \%[3,18]$, which is higher than the incidence of $2 \%$ in our results. However, our findings in general decline on renal function related to TDF exposure were based on a population in which the most patients had preserved kidney function, the pre-existing renal impairment (eGFR $<60 \mathrm{~mL} / \mathrm{min} / 1.73 \mathrm{~m}^{2}$ at baseline) patients in the two studies were $5.2 \%$ and $25.1 \%$ respectively, which are both much higher than $0.1 \%$ of our research.

Of note, a study carried out by Qing Tan et al. revealed that the overall eGFR improved over 24 months following TDF initiation among 269 patients in Chengdu, South China [11]. But, the median eGFR at baseline in their research was higher than that of our study (124.0 vs. $\left.108.2 \mathrm{~mL} / \mathrm{min} / 1.73 \mathrm{~m}^{2}\right)$. Also, Bygrave et al. in South Africa demonstrated that renal function improved in most patients, in which 176 $(18.9 \%)$ had a baseline creatinine clearance $<50 \mathrm{ml} / \mathrm{min}$ [19]. However, their study was highly limited by missing data and lack of key covariates. While, the results of our sensitivity analyses were similar, which were conducted in 5 imputed datasets to reduce bias caused by missing data in our study, making the conclusion of that the overall eGFR decreased throughout the observation period following TDF initiation more robust. Moreover, the decreased trend of eGFR among TDF users was consistent with a previous study conducted among 650 HIV-positive patients with a median eGFR of 108 (IQR: 91-126) $\mathrm{mL} / \mathrm{min} / 1.73 \mathrm{~m}^{2}$ at baseline, which is equivalent to our baseline eGFR level [20].

As in other studies, we found a negative association between stage 3 CKD and baseline eGFR, which was consistent with previous data from South Africa [3]. This cohort focused on individuals with TDF exposure for 48-months revealed that patients with mild (eGFR: $60-89 \mathrm{ml} / \mathrm{min}$; HR 4.8, 95\%Cl: 1.5-15.2) or moderate (eGFR: $30-59 \mathrm{ml} / \mathrm{min}$; HR 15.0, 95\% Cl: 3.4-66.5) renal dysfunction presented a higher risk of nephrotoxicity by 48-months compared to normal renal function. However, the definition of nephrotoxicity in this study was indistinct, which is defined as the occurrence of any decline in kidney function from baseline (acute or chronic) was observed since TDF initiation, which resulted in a lack of precision for the relationship between baseline eGFR and renal dysfunction. Instead, we avoided obscuring associations between the two factors by the clear definition of kidney dysfunction, and the quantification model of the two variables may help clinicians to assess TDF-related CKD risk according to baseline renal function, accurately.

In contrast, the risk of RKFD showed a positive association with baseline eGFR, as also reported by Takeshi and colleagues in Japan [10]. The definition of RKFD in this study was utterly consistent with us. One possible reason for the positive association is that patients with normal renal function show faster responses for TDF exposure compared to patients with renal dysfunction. In our study, the rarely small proportion of patients with kidney impairment conform to this speculation. As in other studies, patients with anemia and higher viral load at baseline were significantly associated with RKFD [21-23]. Such patients deserve particular focus on renal monitoring.

Consistent with previous studies, older age increased the incidence of stage $3 \operatorname{CKD}[11,18,20]$. These findings may reflect the impact of age on TDF circulating levels. Unlike previous studies, we found no 
evidence that lower body mass index (BMI) was independently associated with a higher risk of renal dysfunction. A study that baseline eGFR is equivalent to ours has shown similar findings to ours [20]. These findings may result from unmeasured confounding, although we enrolled in more covariates than others [10] and adjusted for the cohort to account for some of these differences.

Our primary results provided important clinical and research illumination. Treatment with TDF-containing regimen has been widely used as first-line antiretroviral therapy in developing countries; ours is the first largest and well-powered study, which utilized rigorous methodological approaches, to show the risks of TDF-related CKD on a follow-up duration of 6.5 years in China, to our knowledge. Although the rate of stage 3 CKD was low among individuals with TDF exposure, in a country like China with a high quantity of HIV-1 infected patients (1.25 million), which is increasing year by year, the numbers of TDF-related CKD is expected to be high. Therefore, to assess renal function management and monitoring approaches would definitely provide a relevant benefit either for preventing or salvaging TDF nephrotoxicity in these settings; studies like ours underline an urgent need for this topic. Eventually, we identified age and baseline eGFR as important predictors for CKD among HIV-1 infected South Chinese patients receiving TDF. Future studies should focus on whether or not prediction models combined with the two above factors and other variables, such as weight, can effectively help clinicians identify patients at high risk of TDF-related nephrotoxicity, which may alter and improve the therapeutic program.

Other than the limitations mentioned above, the duration of follow-up in this research was short. Longer follow-up will be necessary to assess the long-term impact of TDF on kidney outcomes [24]. The inherent shortcomings of retrospective observational single-center study limited its generality in other populations, and thus further validations from different races are warranted.

To conclude, the present study suggested that a longer duration of TDF using was associated with faster renal function decline, and compared to the low rate of stage 3 CKD in our study, patients infected with HIV-1 mainly experienced RKFD in South China. With the expanded use of TDF, prior to screening for baseline eGFR and taking account of age are necessary to reduce TDF-related CKD before ART initiation. In addition, anemia patients and those with unsuppressed viral load deserve particular focus on renal monitoring. Our results were consistent across a range of sensitivity analyses, and the large sample size makes the results more powerful for HIV-1 positive patients exposed to TDF in China.

\section{Declarations}

\section{Ethics approval and consent to participate}

This study was approved by the Institutional Review Board of Xixi Hospital. All data were anonymized to comply with the provisions of personal data protection legislation. Due to the retrospective nature of this study and due to the fact that only historical medical data were collected, written informed consent was not required.

\section{Consent for publication}


Not applicable.

\section{Availability of data and materials}

The datasets used during the current study are available from the corresponding author on reasonable request.

\section{Competing interests}

The authors declare that they have no competing interests.

\section{Funding}

The study is funded by Hangzhou Agricultural and Social Development Research Project (20201203B191).

\section{Authors' contributions}

LF and JFB conceived, designed, and organized the study, interpreted the results, and drafted the manuscript. JSH helped supervise the study. The other authors contributed to collect the data on site.

\section{Acknowledgments}

We thank all study participants and staff of all participating sites.

\section{References}

1. Nsanzimana S, Remera E, Kanters S, Chan K, Forrest JI, Ford N et al. Life expectancy among HIVpositive patients in Rwanda: a retrospective observational cohort study. The Lancet Global health. 2015;3(3):e169-e77.

2. Smit M, Brinkman K, Geerlings S, Smit C, Thyagarajan K, Sighem Av et al. Future challenges for clinical care of an ageing population infected with HIV: a modelling study. The Lancet Infectious diseases. 2015;15(7):810-8.

3. Brennan A, Evans D, Maskew M, Naicker S, Ive P, Sanne I et al. Relationship between renal dysfunction, nephrotoxicity and death among HIV adults on tenofovir. AIDS (London, England). 2011;25(13):1603-9.

4. Choi Al, Rodriguez RA, Bacchetti P, Bertenthal D, Volberding PA, O'Hare AM. The impact of HIV on chronic kidney disease outcomes. Kidney international. 2007;72(11):1380-7.

5. Kalayjian RC, Lau B, Mechekano RN, Crane HM, Rodriguez B, Salata RA et al. Risk factors for chronic kidney disease in a large cohort of HIV-1 infected individuals initiating antiretroviral therapy in routine care. AIDS (London, England). 2012;26(15):1907-15.

6. Suzuki S, Nishijima T, Kawasaki Y, Kurosawa T, Mutoh Y, Kikuchi Y et al. Effect of Tenofovir Disoproxil Fumarate on Incidence of Chronic Kidney Disease and Rate of Estimated Glomerular 
Filtration Rate Decrement in HIV-1-Infected Treatment-Naïve Asian Patients: Results from 12-Year Observational Cohort. AIDS patient care and STDs. 2017;31(3):105-12.

7. Cihlar T, Ho ES, Lin DC, Mulato AS. Human renal organic anion transporter 1 (hOAT1) and its role in the nephrotoxicity of antiviral nucleotide analogs. Nucleosides, nucleotides \& nucleic acids. 2001;20(4-7):641-8.

8. Scherzer R, Estrella M, Li Y, Choi Al, Deeks SG, Grunfeld C et al. Association of tenofovir exposure with kidney disease risk in HIV infection. AIDS (London, England). 2012;26(7):867-75.

9. Mizushima D, Tanuma J, Kanaya F, Nishijima T, Gatanaga H, Lam NT et al. WHO antiretroviral therapy guidelines 2010 and impact of tenofovir on chronic kidney disease in Vietnamese HIVinfected patients. PloS one. 2013;8(11):e79885.

10. Nishijima T, Kawasaki Y, Tanaka N, Mizushima D, Aoki T, Watanabe K et al. Long-term exposure to tenofovir continuously decrease renal function in HIV-1-infected patients with low body weight: results from 10 years of follow-up. AIDS (London, England). 2014;28(13):1903-10.

11. Tan Q, He Y-H, Yang T-T, Yan D-M, Wang Y, Zhao X et al. Effects of long-term exposure to tenofovir disoproxil fumarate-containing antiretroviral therapy on renal function in HIV-positive Chinese patients. Journal of microbiology, immunology, and infection = Wei mian yu gan ran za zhi. 2019;52(5):710-9.

12. Liu X, Qiu X, Shi C, Huang H, Huang J, Li M et al. Modified glomerular filtration rate-estimating equations developed in asiatic population for chinese patients with type 2 diabetes. International journal of endocrinology. 2014;2014:521071.

13. Chang H, Ye SD. Application of Modified Glomerular Filtration Rate Estimation Equations in Chinese Diabetic Patients with Chronic Kidney Diseases. The West Indian medical journal. 2015;64(3):209-12.

14. Erviti J, Alonso A, Oliva B, Gorricho J, López A, Timoner J et al. Oral bisphosphonates are associated with increased risk of subtrochanteric and diaphyseal fractures in elderly women: a nested casecontrol study. BMJ open. 2013;3(1).

15. White IR, Royston P, Wood AM. Multiple imputation using chained equations: Issues and guidance for practice. Statistics in medicine. 2011;30(4):377-99.

16. Ichikawa M, Hosono A, Tamai Y, Watanabe M, Shibata K, Tsujimura S et al. Handling missing data in an FFQ: multiple imputation and nutrient intake estimates. Public health nutrition. 2019;22(8):135160.

17. Vandenbroucke JP, von Elm E, Altman DG, Gøtzsche PC, Mulrow CD, Pocock SJ et al. Strengthening the Reporting of Observational Studies in Epidemiology (STROBE): explanation and elaboration. International journal of surgery (London, England). 2014;12(12):1500-24. doi:10.1016/j.ijsu.2014.07.014.

18. Msango L, Downs JA, Kalluvya SE, Kidenya BR, Kabangila R, Johnson WD et al. Renal dysfunction among HIV-infected patients starting antiretroviral therapy. AIDS (London, England). 2011;25(11):1421-5. 
19. Bygrave H, Kranzer K, Hilderbrand K, Jouquet G, Goemaere E, Vlahakis N et al. Renal safety of a tenofovir-containing first line regimen: experience from an antiretroviral cohort in rural Lesotho. PloS one. 2011;6(3):e17609.

20. Zachor H, Machekano R, Estrella MM, Veldkamp PJ, Zeier MD, Uthman OA et al. Incidence of stage 3 chronic kidney disease and progression on tenofovir-based regimens. AIDS (London, England). 2016;30(8):1221-8.

21. Ding Y, Duan S, Ye R, Yao S, Cao D, Yang Y et al. Effects of aging, baseline renal function and stage of HIV infection on post-treatment changes in renal function among HIV-infected patients: a retrospective cohort study. HIV medicine. 2019;20(9):591-600.

22. Kimaro FD, Jumanne S, Sindato EM, Kayange N, Chami N. Prevalence and factors associated with renal dysfunction among children with sickle cell disease attending the sickle cell disease clinic at a tertiary hospital in Northwestern Tanzania. PloS one. 2019;14(6):e0218024.

23. Yilma D, Abdissa A, Kæstel P, Tesfaye M, Olsen MF, Girma T et al. Renal function in Ethiopian HIVpositive adults on antiretroviral treatment with and without tenofovir. BMC infectious diseases. 2020;20(1):582.

24. Mocroft A, Lundgren JD, Ross M, Fux CA, Reiss $P$, Moranne $O$ et al. Cumulative and current exposure to potentially nephrotoxic antiretrovirals and development of chronic kidney disease in HIV-positive individuals with a normal baseline estimated glomerular filtration rate: a prospective international cohort study. The lancet HIV. 2016;3(1):e23-e32.

\section{Tables}

Table 1 Characteristics at cohort entry 


\begin{tabular}{|c|c|}
\hline Characteristic & No. (\%) or Median (IQR) \\
\hline Age (years) & $31(26-39)$ \\
\hline Female & $45(5.7 \%)$ \\
\hline Weight (kg) & $63(57-69)$ \\
\hline Height $(\mathrm{cm})$ & $172(168-175)$ \\
\hline BMI $\left(\mathrm{kg} / \mathrm{m}^{2}\right)$ & $21.2(19.6-23.0)$ \\
\hline CD4 (cells/ $\mu \mathrm{L})$ & $272(151-397)$ \\
\hline Triglycerides (mmol/L) & $1.2(0.9-1.7)$ \\
\hline Total cholesterol (mmol/L) & $3.9(3.4-4.5)$ \\
\hline eGFR $\left(\mathrm{mL} / \mathrm{min}\right.$ per $\left.1.73 \mathrm{~m}^{2}\right)$ & $108.2(96.8-120.8)$ \\
\hline Dyslipidemia & $231(29.6 \%)$ \\
\hline \multicolumn{2}{|l|}{ Risk factor } \\
\hline Homosexual & $580(72.8 \%)$ \\
\hline Heterosexual & $204(25.6 \%)$ \\
\hline Injection drug user & $1(0.1 \%)$ \\
\hline Other & $12(1.5 \%)$ \\
\hline WHO stage III/IV & $276(34.6 \%)$ \\
\hline \multicolumn{2}{|l|}{ Hepatitis B status } \\
\hline Positive & $103(12.9 \%)$ \\
\hline Negative & $616(77.3 \%)$ \\
\hline Unknown & $78(9.8 \%)$ \\
\hline \multicolumn{2}{|l|}{ Hepatitis C status } \\
\hline Positive & $16(2.0 \%)$ \\
\hline Negative & $685(86.0 \%)$ \\
\hline Unknown & $96(12.0 \%)$ \\
\hline Anemia & $90(11.4 \%)$ \\
\hline Diabetes & $51(6.5 \%)$ \\
\hline \multicolumn{2}{|l|}{ Viral load (copies per mL) } \\
\hline$<400$ & 68 (8.5\%) \\
\hline
\end{tabular}




\begin{tabular}{|ll|}
\hline$>=400,<10000$ & $192(24.1 \%)$ \\
\hline$>=10000,<100000$ & $195(24.5 \%)$ \\
\hline$>=100000$ & $84(10.5 \%)$ \\
\hline Unknown & $258(32.4 \%)$ \\
\hline Viral load (log10 per $\mathrm{mL})$ & $4.1(3.4-4.7)$ \\
\hline Renal dysfunction & $1(0.1 \%)$ \\
\hline Protease inhibitors exposure (LPV/r) & $10(1.3 \%)$ \\
\hline AIDS diagnosis days & $23(13-81)$ \\
\hline
\end{tabular}

Data are No. (\%) or median (IQR) unless otherwise indicated. Baseline was defined as the date of starting antiretroviral therapy on or after January, 2010. Diabetes and dyslipidemia were defined by diagnosis or related medication. Anemia was defined as hemoglobin $<12.0 \mathrm{~g} / \mathrm{dL}$ in women and $<13.0 \mathrm{~g} / \mathrm{dL}$ in men. Coinfection with hepatitis $B$ defined by positive hepatitis B surface antigen, coinfection with hepatitis $C$ defined by positive HCV viral load. Renal dysfunction referred to baseline eGFR $<60 \mathrm{~mL} / \mathrm{min}$ per1.73 $\mathrm{m}^{2}$.

Abbreviations: IQR, interquartile range; BMI, body mass index; eGFR, estimated glomerular filtration rate; WHO, World Health Organization; LPV, lopinavir; AIDS, acquired immune deficiency syndrome.

Table 2 Predicted eGFR change rates in the general linear mixed-effects regression model

\begin{tabular}{|lllll|}
\hline Cumulative Exposure & Model 1 & \multicolumn{3}{l|}{ Model 2 } \\
\cline { 2 - 5 } & $\operatorname{Exp}(\beta)^{\mathrm{a}}(95 \% \mathrm{Cl})$ & $P$ value & $\operatorname{Exp}(\beta)^{\mathrm{a}}(95 \% \mathrm{Cl})$ & $P$ value \\
\hline Per 48 weeks ${ }^{\mathrm{b}}$ & $-1.22(-1.60,-0.88)$ & $<0.01$ & $-1.29(-1.84,-0.75)$ & $<0.01$ \\
\hline$<48$ weeks & $-2.80(-3.69,-1.91)$ & $<0.01$ & $-2.59(-3.48,-1.71)$ & $<0.01$ \\
\hline $48-96$ weeks & $-4.09(-5.28,-2.91)$ & $<0.01$ & $-3.65(-4.86,-2.45)$ & $<0.01$ \\
\hline $96-144$ weeks & $-4.07(-5.42,-2.72)$ & $<0.01$ & $-3.64(-5.07,-2.21)$ & $<0.01$ \\
\hline $144-192$ weeks & $-5.28(-6.79,-3.78)$ & $<0.01$ & $-4.88(-6.53,-3.23)$ & $<0.01$ \\
\hline $192-240$ weeks & $-9.62(-11.98,-7.26)$ & $<0.01$ & $-8.89(-11.39,-6.39)$ & $<0.01$ \\
\hline $240-288$ weeks & $-13.80(-18.47,-9.12)$ & $<0.01$ & $-13.23(-18.04,-8.41)$ & $<0.01$ \\
\hline$\geq 288$ weeks & $-18.35(-20.98,-15.73)$ & $<0.01$ & $-17.61(-21.09,-14.14)$ & $<0.01$ \\
\hline
\end{tabular}

Model 1: adjusted for age, sex and BMI at baseline.

Model 2: adjusted for age, sex, BMI, CD4 count, eGFR, dyslipidemia, HIV/AIDS risk factors, WHO stage III/IV HIV/AIDS, hepatitis B positivity, hepatitis C positivity, anemia, diabetes, HIV-1 RNA viral load, 
lopinavir exposure at baseline and AIDS diagnosis days before TDF initiation.

${ }^{a}$ Loss in eGFR on the different duration of TDF exposure.

${ }^{b}$ Mean loss in eGFR $\left(\mathrm{mL} / \mathrm{min} / 1.73 \mathrm{~m}^{2}\right)$ per 48 weeks.

Figures

\section{Fig. 1}

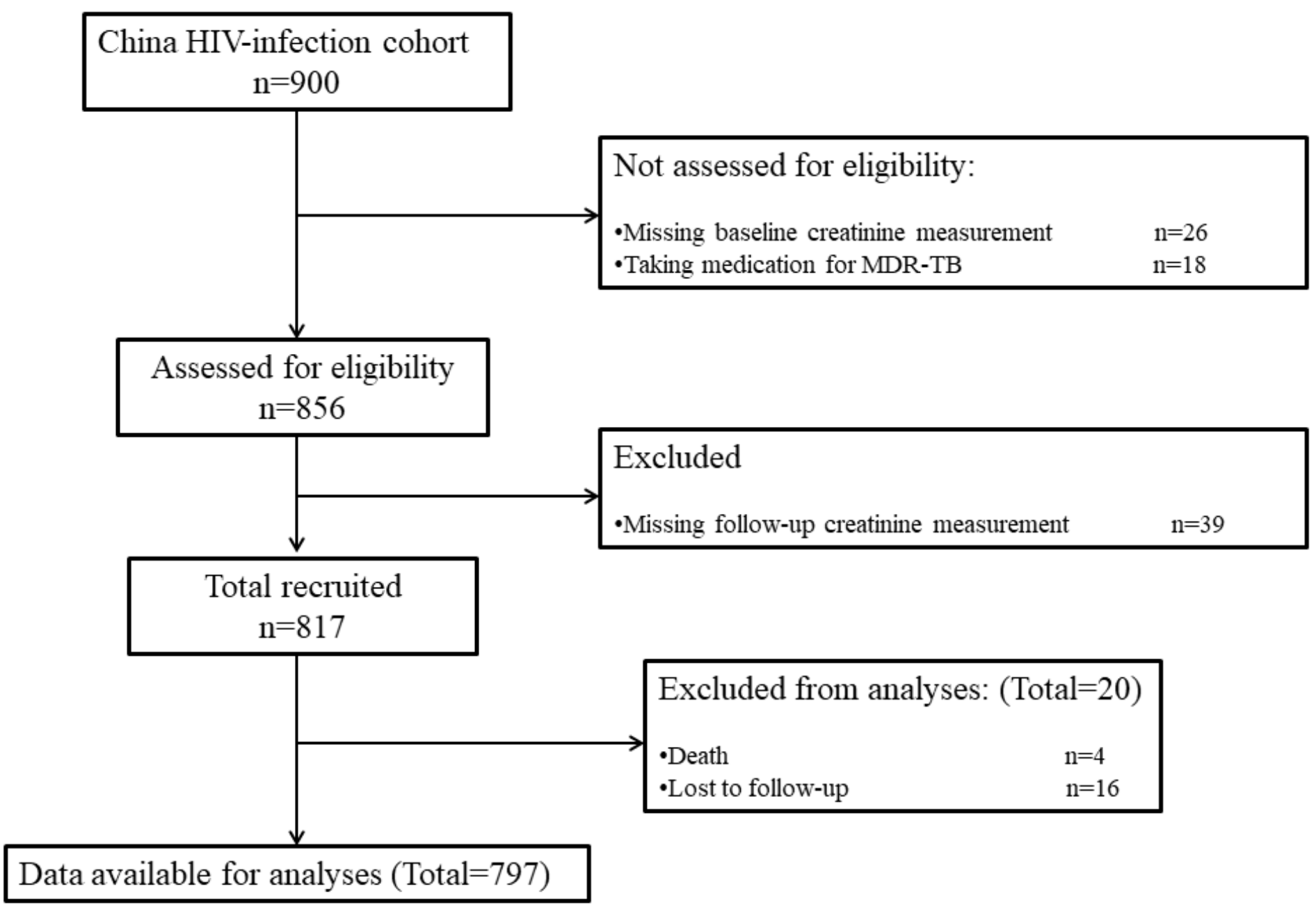

Figure 1

Study selection flow Flowchart showing the study selection among TDF users infected HIV-1. 
Fig. 2

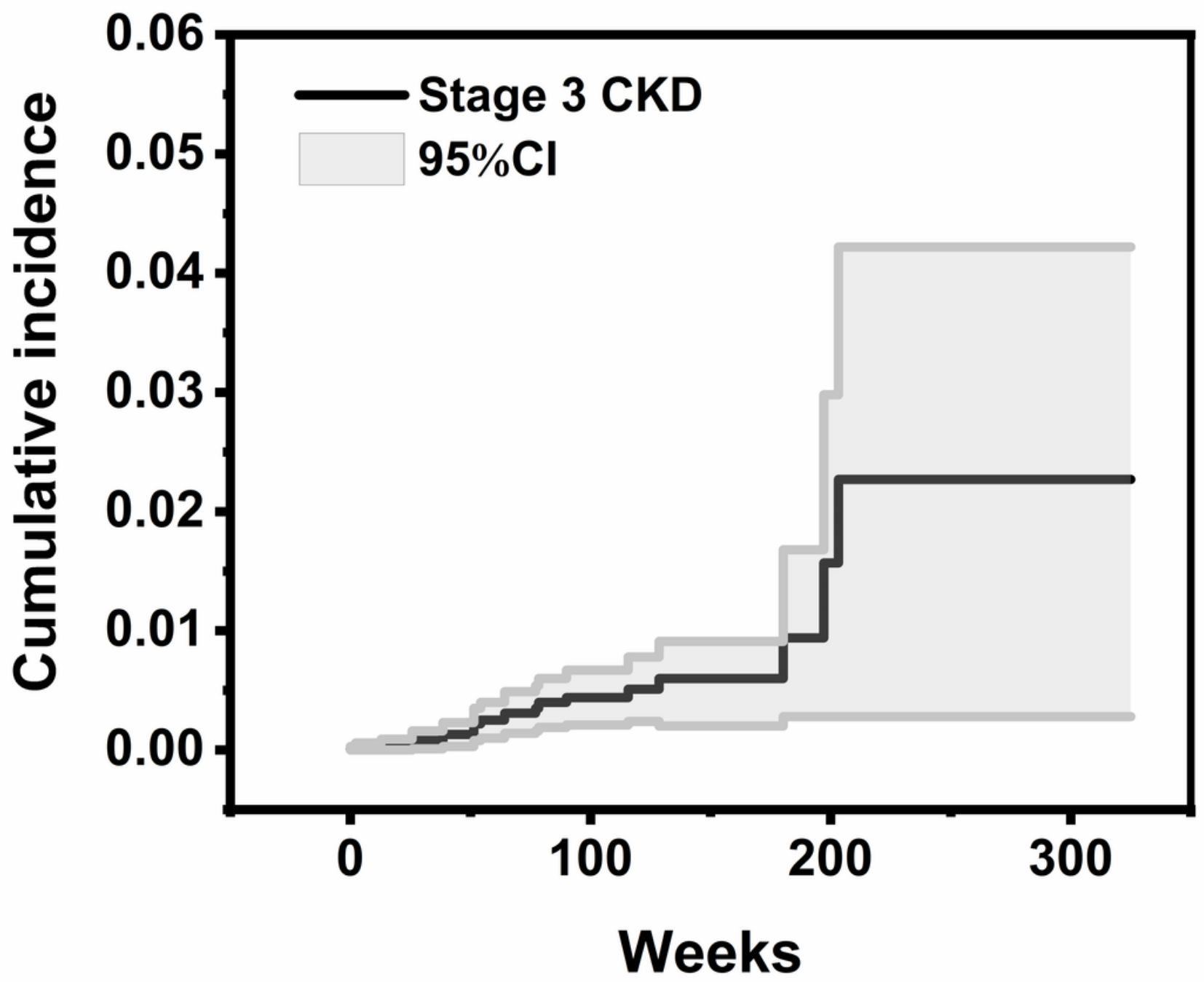

Figure 2

Kaplan-Meier graph of cumulative incidence of stage 3 CKD Outcome defined as eGFR $\geq 60$ $\mathrm{mL} / \mathrm{min} / 1.73 \mathrm{~m} 2$ at baseline and eGFR $<60 \mathrm{~mL} / \mathrm{min} / 1.73 \mathrm{~m} 2$ during follow-up period. Abbreviations: CKD, chronic kidney disease; eGFR, estimated glomerular filtration rate. 


\section{Fig. 3}

Characteristics

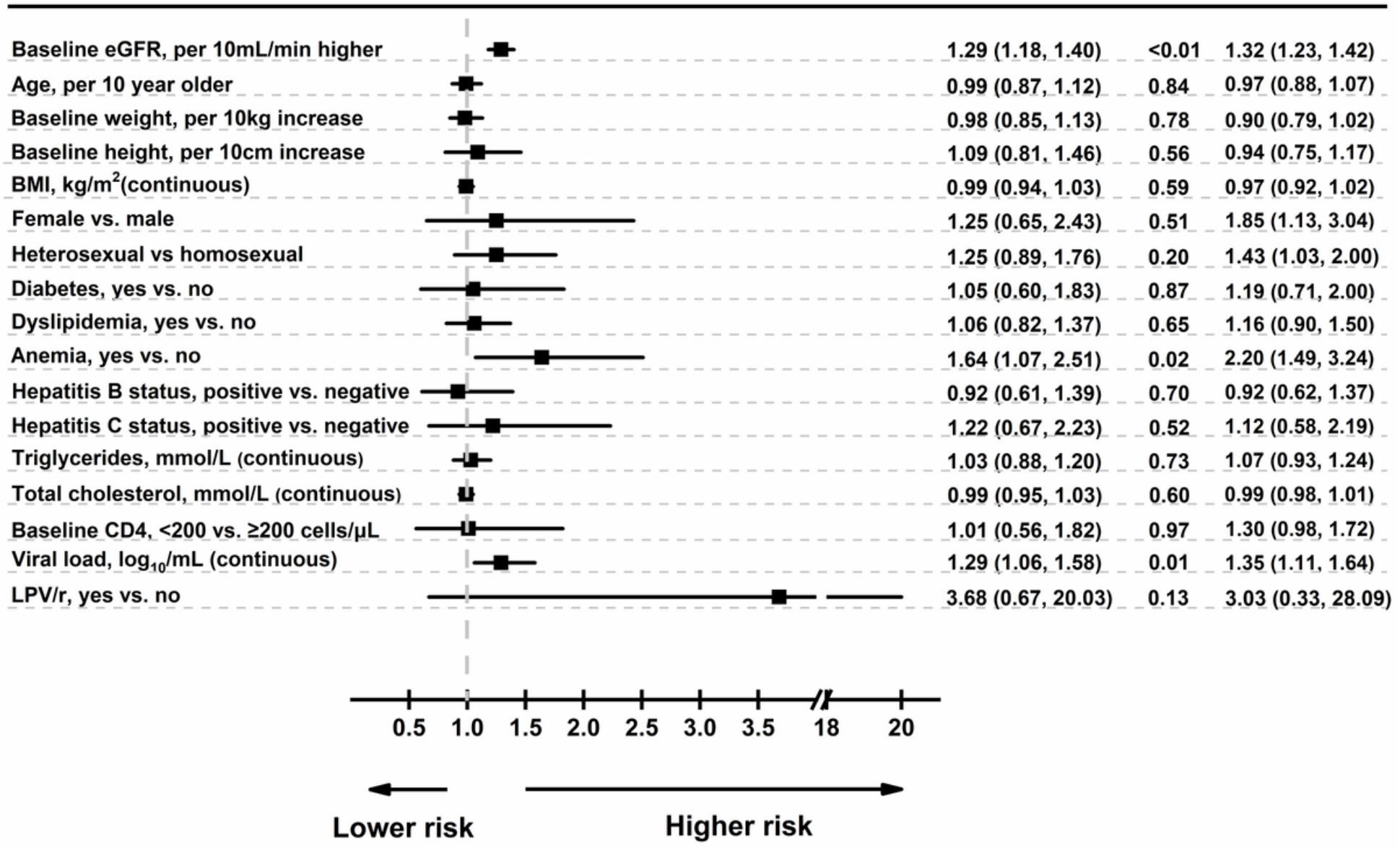

\section{Figure 3}

Determinants of reduced kidney function: multivariate analysis using Generalized Estimate Equation Model a Adjusted for age, sex and BMI at baseline. b Adjusted for age, sex, BMI, CD4 count, eGFR, dyslipidemia, HIV/AIDS risk factors, WHO stage III/IV HIV/AIDS, hepatitis B positivity, hepatitis C positivity, anemia, diabetes, HIV-1 RNA viral load, lopinavir exposure at baseline and AIDS diagnosis days before TDF initiation. Abbreviations: aOR, adjusted odds ratio; $\mathrm{COR}$, crude odds ratio; eGFR, estimated glomerular filtration rate; BMI, body mass index; WHO, World Health Organization; LPV, lopinavir. 


\section{Fig. 4}

Characteristics

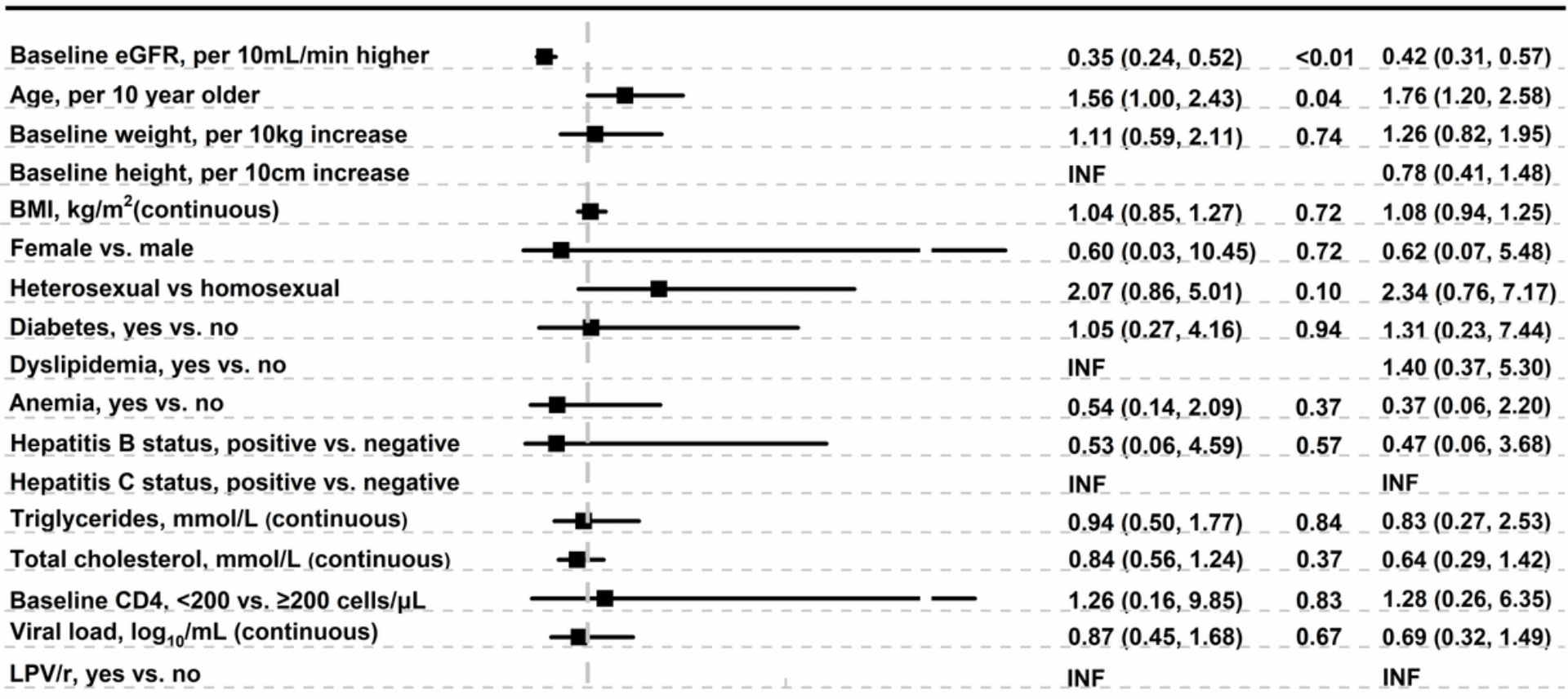

$\operatorname{aOR}^{\mathrm{a}}(95 \% \mathrm{CI}) \quad P$ values $\operatorname{cOR}^{\mathrm{b}}(95 \% \mathrm{CI})$

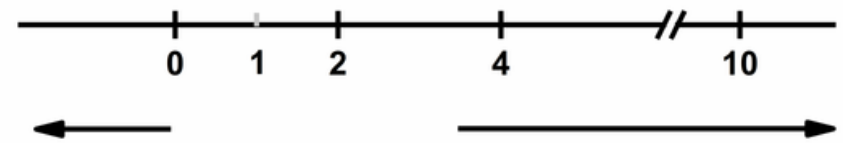

Lower risk

\section{Figure 4}

Determinants of stage 3 CKD: multivariate analysis using Generalized Estimate Equation Model a Adjusted for age, sex and BMI at baseline. b Adjusted for age, sex, BMI, CD4 count, eGFR, dyslipidemia, HIV/AIDS risk factors, WHO stage III/IV HIV/AIDS, hepatitis B positivity, hepatitis C positivity, anemia, diabetes, HIV-1 RNA viral load, lopinavir exposure at baseline and AIDS diagnosis days before TDF initiation. Abbreviations: aOR, adjusted odds ratio; $\mathrm{COR}$, crude odds ratio; eGFR, estimated glomerular filtration rate; BMI, body mass index; WHO, World Health Organization; LPV, lopinavir; INF, infinite.

\section{Supplementary Files}

This is a list of supplementary files associated with this preprint. Click to download.

- Additionalfile1.docx 